\title{
Characterization of myocardial deformation in patients with different etiologies of left ventricular hypertrophy by using strain distribution from Magnetic Resonance Imaging
}

\author{
Gemma Piella ${ }^{1,2 \S}$, Mathieu De Craene ${ }^{1,2}$, Bart H. Bijnens ${ }^{3,1,2}$, Catalina Tobon-Gomez ${ }^{1,2,4}$, \\ Marina Huguet ${ }^{4,2}$, Gustavo Avegliano ${ }^{5}$, Alejandro F. Frangi ${ }^{1,2,3}$ \\ ${ }^{1}$ Center for Computational Imaging and Simulation Technologies in Biomedicine (CISTIB), Departamento de \\ Tecnologías de la Información y las Comunicaciones, Universitat Pompeu Fabra, Barcelona, Spain; \\ ${ }^{2}$ Networking Biomedical Research Center on Bioengineering, Biomaterials and Nanomedicine (CIBER-BBN), \\ Barcelona, Spain; \\ ${ }^{3}$ Institució Catalana de Recerca i Estudis Avançats (ICREA), Barcelona, Spain; \\ ${ }^{4}$ CETIR Sant Jordi, Barcelona, Spain \\ ${ }^{5}$ Instituto Cardiovascular de Buenos Aires, Argentina
}

This work was partially funded by the Industrial and Technological Development Centre (CDTI) under the CENIT Programme (CDTEAM project) and the European Community’s Seventh Framework Programme (FP7/2007-2013) [grant number 224495] (euHeart project). G. Piella was supported by the Ramón y Cajal Programme from the Spanish Ministry of Science and Innovation. 


\section{Abstract}

\section{Introduction and objectives}

It has been suggested that in hypertrophic cardiomyopathy (HCM) the regional fibre disarray results in segments of none or severely reduced deformation, distributed non-uniformly within the left ventricle (LV). This is in contrast with other types of hypertrophies such as athlete's heart or hypertensive left ventricular hypertrophy (HT-LVH) which may have abnormal cardiac deformation but never as reduced as showing absence of deformation in certain segments. Hence, we propose to use the distribution of the strain values to study deformation in HCM.

\section{Methods}

Using tagged magnetic resonance imaging, we reconstructed the LV systolic deformation from 12 controls, 10 athletes, 12 patients with HCM and 10 patients with HT-LVH. Deformation was quantified using a fast nonrigid registration algorithm and measuring radial and circumferential peak systolic strain values from $16 \mathrm{LV}$ segments.

\section{Results}

HCM patients showed significantly lower average strain values when compared to other groups. However, while the deformation in healthy subjects and HT-LVH was concentrated around the mean value, in HCM there was a coexistence of segments with normal contraction and segments with none or significantly reduced deformation, resulting in a larger heterogeneity of the strain values. Some non-deforming segments were also found in the absence of fibrosis or hypertrophy.

\section{Conclusions}

Strain distribution characterizes specific patterns of myocardial deformation in patients with different etiologies of LVH. HCM patients had significantly lower average strain as well as 
larger strain heterogeneity (compared to controls, athletes and HT-LVH), and they

presented non-deforming regions.

Key words: left ventricular hypertrophy, hypertrophic cardiomyopathy, strain distribution, tagged magnetic resonance imaging

\section{List of abbreviations}

HCM: hypertrophic cardiomyopathy

HT-LVH: hypertensive left ventricular hypertrophy

LV: left ventricle

LVH: left ventricle hypertrophy

MRI: magnetic resonance imaging

LGE: late gadolinium enhancement 


\section{Introduction}

Hypertrophic cardiomyopathy (HCM) is a genetic disorder characterized by a hypertrophied non-dilated left ventricle (LV) with regions of myocardial fiber disarray. HCM is normally identified by an increased LV wall thickness, usually quantified by echocardiography or magnetic resonance imaging (MRI). The distribution of the hypertrophy may take many forms and vary greatly among patients but it is usually most prominent in the ventricular septum. In addition, the absolute thickness of the wall may also differ greatly: while normal thickness of the LV is $12 \mathrm{~mm}$ or less; in HCM the thickness is usually $15 \mathrm{~mm}$ or more (up to extremely thick walls of more than $30 \mathrm{~mm}$ ). It is known, however, that people with a mutant HCM gene may have normal wall thickness ${ }^{1}$. Moreover, excessive thickening of the heart muscle may occasionally occur as part of other diseases, such as mitochondrial disorders or Fabry's disease ${ }^{1}$.

Besides HCM, other examples of left ventricular thickening (i.e. hypertrophy) are hypertensive left ventricular hypertrophy (HT-LVH) and athlete's heart (physiological remodeling). All these forms can present overlapping cardiac hypertrophy phenotypes as determined by conventional echocardiography ${ }^{2,3}$. Ultrasound tissue velocities and deformation analysis have been lately introduced to study and to distinguish HCM from other forms of left ventricular hypertrophy $(\mathrm{LVH})^{4-6}$.

In HCM, even if global ventricular function appears normal, regional myocardial function and long-axis motion are significantly impaired. All studies quantifying myocardial deformation in HCM conclude that regional deformation is overall decreased compared to normal subjects ${ }^{7-9}$. Traditionally, using either tagged MRI or ultrasound strain imaging, the

global or regional strains averaged over the whole population is calculated. However, it has been suggested that HCM (as opposed to HT-LVH or athlete's heart) is associated with 
regions of absent or severely reduced deformation while other segments show normal strains $^{5}$. This results in an overall decrease when average strain is calculated. In order to maintain the diagnostic information from the inhomogeneous amplitude and spatial location of the regional deformation in HCM, we propose to calculate the distribution of the strain values from tagged MRI of the whole LV. Comparing the distributions from pathological and normal ventricles should easily identify both the overall decrease in deformation and the presence of segments with abnormal strain.

The purpose of the present study was to characterize regional systolic deformation measured using tagged MRI and a nonrigid registration approach in HCM and to compare it with other forms of LVH such as HT-LVH and athlete's heart.

\section{Methods}

\section{Study population and data acquisition}

In total, 44 subjects were included in the study. From these, 12 (8 male, aged $61.4 \pm 14.1$ years) were diagnosed with HCM (with 4 obstructive and 8 non-obstructive asymmetrical

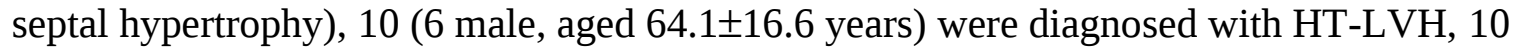
were healthy trained cyclist athletes (10 male, aged 23.2 \pm 3.0 years) and 12 were healthy sedentary controls (8 male, aged $28.7 \pm 2.7$ years). The diagnosis of HCM was made by echocardiography in the absence of other causes as systemic hypertension or aortic stenosis which could lead to LVH. The diagnosis of HT-LVH was based on the evidence of concentric LVH by echocardiography and sustained increased in systolic blood pressure (above $140 \mathrm{mmHg}$ ) and/or diastolic blood pressure (above $90 \mathrm{mmHg}$ ), in the absence of other causes of secondary hypertension. In all cases, the diagnosis of LVH was confirmed 
by MRI. Baseline characteristics of the studied population are listed in Table 1 . The study complied with the declaration of Helsinki and informed consent was obtained from each participant.

Imaging was performed in breath-hold using a General Electric Signa CVi-HDx, 1.5 T scanner (General Electric, Milwaukee, USA). A SPAMM grid tag pattern was applied starting at end-diastole. The slice thickness was $8 \mathrm{~mm}$ with an in-plane resolution of 0.78 $\mathrm{mm} \times 0.78 \mathrm{~mm}$ and no gaps between slices. The tagging flip angle was $20^{\circ}$ with tag spacing of $6 \mathrm{~mm}$ in the vertical and horizontal direction. Thirty phases were reconstructed per cardiac cycle; each one comprising 8 to 10 slices in short-axis and 3 to 6 slices in long-axis covering the whole of the LV. For some of the sequences (24 of the 38) long-axis images were acquired as parallel planes while the rest were acquired as three radial planes with tag plane orientations of $0^{\circ}, 45^{\circ}$ and $90^{\circ}$ around the $\mathrm{LV}$ central axis.

The first phase corresponded to end-diastole, and end-systole was defined as the phase with minimal intraventricular area (visually estimated from the tagged images). The number of systolic phases varied from subject to subject but it was approximately one third of the cardiac cycle. For the registration and deformation analysis, only the systolic phases plus one diastolic phase were used since the tags faded to undetectable levels by early diastole (thus making it not possible to track myocardial deformation from that phase on). Additionally, for HCM patients, late gadolinium enhancement (LGE) MRI was obtained 10 minutes after injection of $0.2 \mathrm{mmol} / \mathrm{kg}$ IV of gadopentate dimeglumine contrast. The LGE images were acquired using an inversion recovery sequence with $8 \mathrm{~mm}$ slice thickness at the same position as the short-axis tagged images in end-diastole. 


\section{Data analysis}

The strain tensor was computed from tagged MRI sequences applying a nonrigid

registration algorithm ${ }^{10,11}$. When parallel long-axis views were available, both short-axis and long-axis views were used ${ }^{10}$. Through registration, the correspondences between the myocardial points in end-diastole and the myocardial points in each of the systolic phases were found.

To reduce the high computational cost of registration, we used a two-level acceleration strategy where registrations of consecutive images in the sequence are distributed over different multiprocessor nodes of a grid computing infrastructure and, within each node, the process is optimized by using different parallelization techniques ${ }^{11,12}$. This acceleration strategy allowed noticeably speeding up the registration process and lowering the strain computation times to manageable values (approximately 1 minute instead of 65 minutes per patient). The grid infrastructure consisted of a 24-node SGI Altix ICE 8200 cluster, each node running two quad-core Intel Xeon (2.66 GHz CPU, 16 GB RAM) processors, thus in total 192 CPUs.

The strain tensor was then obtained from the analytical expression of the spatial gradient of the displacement field (obtained by the registration process) and projected onto the radial and circumferential directions corresponding to the geometry of the heart at end-diastole (i.e., the undeformed state). Negative values indicate shortening (or thinning), and positive values indicate lengthening (or thickening). For each direction and each time frame, the strain data was averaged into 16 regions (apex excluded) in accordance with the standard segmentation proposed by the American Heart Association ${ }^{13}$. Peak strain was determined as the maximum strain magnitude on the strain curve. Subsequently, the distribution 
(histogram) of the peak strain values was obtained by considering the location-independent frequency of the segmental peak strain values.

Myocardial fibrosis was assessed by LGE. The LGE image set was reviewed by 2 blind observers for the presence of hyperenhancement in each myocardial segment of the HCM patients. This hyperenhancement reveals areas of increased interstitial space in the myocardium, which in the case of HCM is likely due to myocardial fibrosis.

\section{Statistical Analysis}

The empirical strain distributions were compared with a Gaussian curve. Some of them showed a slight deviation from normal distribution. These observations were supported by performing the Shapiro-Wilk test for normality. The homogeneity of variance among different groups was tested using both the Levene's test and the Brown-Forsythe test. We concluded from these tests that not all variances could be assumed equal. Hence, comparisons between different study groups were done using a Welch's test (i.e., unequal variance t-test) which is robust even if the distributions are only approximately normal. Dispersion of strain (non-normal with variance homogeneity data) was compared between groups with a Mann-Whitney U test. Due to the descriptive exploratory character of the present study no adjustment for multiple comparisons was made. Results were expressed as mean \pm standard deviation, and the differences were considered statistically significant at a two-sided $p$ value less than 0.05 . The current study had adequate power (above $90 \%$ ) to detect statistically significant differences in strain values. 


\section{Results}

Some individual segments (14 in the normal volunteers, 9 in the HCM patients and 6 in the HT-LVH patients) corresponding mostly to the apical level were excluded from the statistical analysis due to the poor quality of the images (low tag contrast which led to registration artifacts). Thus, a total of 675 (95.9\%) segments out of 704 were evaluated.

Tables 2 and 3 present the average radial and circumferential peak systolic strains in each segment for all studied groups.

Controls showed a regional strain pattern in agreement with previous studies ${ }^{14-16}$. Radial strain was highest in the anterior and lateral wall. Circumferential strain varied more predictably (less variance), tending to be greatest in the septum and gradually increasing from base toward the apex.

The athletes had radial strain decreased in almost all myocardial segments compared with controls, especially in the anterior and lateral walls. Circumferential strain was equal or slightly less than controls except for anterior and anterolateral regions at mid and apical levels where it increased. Globally, radial strain was lower (17.4 \pm 3.9 vs $22.7 \pm 4.5 \%$; $p=0.0007$ ) whereas circumferential strain showed no significant difference with respect to controls (-13.6 \pm 2.9 vs $-13.6 \pm 2.2 \% ; p=0.38)$.

HCM patients had significantly reduced strain values in all 16 segments when compared to other groups. The radial strain was generally larger at the lateral wall than at the septum. Circumferential strain was particularly diminished at inferior and inferoseptal regions basal and midventricular level, and in the septum and lateral wall at the apical level. On average, they presented a lower mean with larger deviation than all other groups: compared to controls (13.8 $\pm 5.2 \%$ vs $22.7 \pm 4.5 \%$; $p<0.0001$ in the radial direction, and $-9.2 \pm 3.4 \%$ vs 
$-13.6 \pm 2.2 \% ; p<0.0001$ in the circumferential direction), compared to athletes $(13.8 \pm 5.2 \%$ vs $17.4 \pm 3.9 \% ; p<0.0001$ in the radial direction, and $-9.2 \pm 3.4 \%$ vs $-13.6 \pm 2.9 \% ; p<0.0001$ in

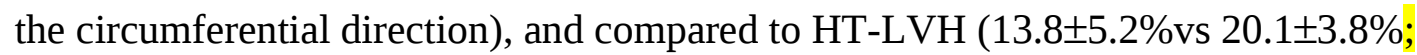

$p<0.0001$ in the radial direction, and $-9.2 \pm 3.4 \%$ vs $-11.3 \pm 3.1 \%$; $p=0.04$ in the circumferential direction).

HT-LVH patients showed strain reduction compared to controls (20.1 $33.8 \%$ vs $22.7 \pm 4.5 \%$;

$p=0.09$ in the radial direction, and $-11.3 \pm 3.1 \%$ vs $-13.6 \pm 2.2 \%$; $p=0.01$ in the circumferential direction). Differences were especially marked in the septum at the basal and medial levels, while all other segments did not differ significantly.

Figure 1 shows for every study group the distribution of the radial (left) and circumferential (right) peak systolic strain considering all the evaluated segments independently of their location. Each distribution curve shows the proportion of segments (vertical axis) over the range of strain values observed (horizontal axis). From Figure 1, it is clear that while the deformation in controls, athletes and HT-LVH is concentrated around the mean value, in HCM there is a coexistence of segments with normal contraction and segments with absent or significantly reduced deformation, resulting in a larger variability of the values.

Figure 2 shows the boxplots of the strain distributions. In each box, the central mark is the median, the edges of the box are the first and third quartiles, the whiskers extend to the most extreme data values without considering outliers, and the outliers are plotted individually as crosses.

Tables 4 and 5 compare the dispersion (within a group) of radial and circumferential peak strain values. Variability of strain within each group of study was quantified by measuring the coefficient of variation (ratio of standard deviation to the absolute mean value), range 
(difference between highest and lowest value) and inter-quartile range (difference between the third and first quartiles) of each individual's strain distribution, and averaging within each group. It should be pointed out, however, that the coefficient of variation (CV) is a more appropriate way of measuring dispersion than range or inter-quartile range (IQR) since data sets with different mean values are being compared. Radial dispersion (Table 4) was greater in controls and HCM patients than in athletes or HT-LVH patients. HCM patients presented a larger coefficient of variation $(29.7 \pm 5.3 \%$ vs $21.0 .0 \pm 2.2 \%$; $p<0.0001)$ than controls, but lower range (15.8 \pm 3.8 vs $17.7 \pm 1.5 ; p=0.004)$. There were no significant differences for inter-quartile range between HCM and controls. Circumferential dispersion (Table 5) was significantly larger in HCM group than in any other.

In HCM patients we could identify segments of very low deformation, while such segments were not present in either the controls, athletes or HT-LVH (where the strain values were always above $9 \%$ for the radial direction and below $-6.5 \%$ for the circumferential direction). Moreover, the presence of at least one segment with extremely low deformation (below 3.5 standard deviations from the controls) discriminated HCM from HT-LVH and athlete's heart with a sensitivity of $84 \%$ and a specificity of $100 \%$ when using the radial strain, and a sensitivity of $100 \%$ and a specificity of $100 \%$ when using the circumferential strain. This discrimination was not possible when looking only at the global average strain. Using the CV, ROC analysis for discrimination between HCM and HT-LVH showed the optimal cutoff value for radial CV to be $24.7 \%$ (sensitivity $91.6 \%$, specificity $85.7 \%$, accuracy $89.5 \%$ ) and for circumferential CV $24.5 \%$ (sensitivity $75 \%$, specificity $100 \%$, accuracy $84.2 \%$ ). For HCM patients, we also evaluated the relationship between non-deforming segments (assessed by strain analysis), maximum end-diastolic myocardium wall thickness and 
fibrosis (assessed by LGE imaging). LGE occurred in 8 (67\%) of the 12 patients with HCM. More specifically, it occurred in 30 (16.4\%) of the 183 analysed segments, being predominant in the most hypertrophied regions and most often in the antero-septal and infero-septal segments. Out of the 30 LGE segments, 18 (60\%) were non-deforming segments. This is in contrast with the non LGE segments from which only 18 (11.8\%) out of 153 segments were non-deforming. These results are summarized in Table 6 . The relation between LGE and maximum wall thickness is shown in Figure 3. In general, patients with positive LGE had higher maximum end-diastolic wall thickness $(21.1 \pm 3.1$ $\mathrm{mm})$ than patients with negative LGE $(18.2 \pm 2.5 \mathrm{~mm})$, but the differences were not statistically significant $(\mathrm{p}=0.1)$. Non-deforming segments were found in $36(19.7 \%)$ of the 183 analysed segments. They had a non-uniform distribution although they were more frequently $(69 \%)$ detected in the septum and hence, since the HCM patients had septal asymmetrical hypertrophy, within the thickest myocardial segments. Among the non-deforming segments, LGE was detected in $18(50 \%)$ of the 36 segments. In contrast, from the 147 deforming segments, only 12 (8.2\%) showed LGE. These results are shown in Table 7.

\section{Discussion}

While all forms of LVH show thickening of the myocardium, each of the different etiologies has a specific signature in regional deformation changes. Our results, along with previous studies, confirm that regional deformation is impaired in LVH and provide information about the heterogeneity of the dysfunctional regions.

HCM is histologically characterized by myocyte disarray resulting in local dysfunctional myocardium and potentially abnormal increase of fibrotic tissue ${ }^{17,18}$. Kim et al. 
${ }^{19}$ demonstrated that, regardless of myocardial hypertrophy, the presence of regional fibrosis is associated with decreased (circumferential) strain. It has also been shown that regional function is decreased in association with the degree of hypertrophy ${ }^{7,20}$. Thus, the regional variation in the myocardial disarray and fibrosis may explain the heterogeneity regional function that has been frequently reported in $\mathrm{HCM}^{7,9,19,20}$. Our results showed that the HCM patients had significantly reduced strain values in all 16 segments even in the absence of fibrosis or hypertrophy. Circumferential strain was noticeably reduced at inferior and inferoseptal wall at basal and midventricular level, and in the septum and lateral wall at apical level. These results are in agreement with previously reported studies ${ }^{7-9}$. The coexistence of segments with normal contraction and segments with absent or significantly reduced deformation explains the larger deviation of HCM with respect to the other study groups. Depending on the particular patient, these non-deforming regions were located anywhere within the LV, but they were most often localized in the septum, while in the lateral wall, deformation was usually normal. These findings are consistent with those by Orlando et al. ${ }^{5}$ in which HCM was shown to be characterized by the presence of nondeforming segments (detected by ultrasound strain imaging) distributed non-uniformly in the LV.

Non-deforming segments were present in all HCM patients, most often in the interventricular septum (69\%) and among LGE segments (60\%) but without being indicative of fibrosis (only 50\% of the non-deforming segments showed LGE). Dually, although the occurrence of fibrosis was significantly higher in non-deforming segments (50\%) than in segments which deform (8.2\%), the presence of LGE was not an accurate indicator of absence of deformation. These results suggest that absence of deformation is not systematically a response to fibrosis or hypertrophy (nor indeed is fibrosis or 
hypertrophy a response to absence of deformation). Hence, the presence of fibrosis or hypertrophy cannot always identify deformation abnormalities.

HT-LVH is characterized by increased LV mass and relative wall thickness ${ }^{21}$. It is often associated with concentric (symmetrical) hypertrophy and reduced global systolic function due to long-standing pressure overload ${ }^{22}$. Further studies have shown that there is a significant reduction in longitudinal deformation in the basal septum ${ }^{5,22}$. In our study, we found that the HT-LVH patients had slightly reduced global radial and circumferential strain when compared to normals, with the most significant reduction in the inferoseptal and anteroseptal segments at the basal level. However, while overall decreased deformation was present, strain values were never as low as showing absence of deformation as observed in HCM. This agrees with previous studies ${ }^{5}$. We also found that HT-LVH patients showed dispersion in radial strain similar to controls while dispersion in circumferential strain was larger. For both radial and circumferential, dispersion in HT-LVH patients was substantially less than that found for HCM patients.

Intensive regular training leads to several morphological and functional modifications in the heart. The athlete's heart refers to the LV adaptation to long-term intensive endurance training characterized by an increase in chamber size, wall thickness and mass ${ }^{23}$. However, these changes may also be an early sign of cardiac diseases for those who have inherited tendencies to develop enlarged and thickened hearts, hypertrophic cardiomyopathies, or similar abnormalities. Thus, differential diagnosis between pathological and physiologic (non-pathological) responses is critical. Peterson et al. ${ }^{24}$ proposed to use MRI-derived LV volume and geometric indices to distinguish athlete's heart from pathological forms of LVH. These indices, however, could not discriminate HCM from HT-LVH. On the other hand, there are few studies on regional myocardial strains for endurance athletes and 
sometimes contradictory results had been reported. A study by Richand $e t a l{ }^{25}$ using ultrasound speckle tracking showed that physiologic hypertrophy that occurs in elite athletes can be distinguished from pathological hypertrophy using myocardial deformation analysis. They found that radial and circumferential strains were significantly higher in athletes compared to controls and HCM patients. Recently, a study by Nottin et al. ${ }^{26}$ using tissue Doppler showed that apical radial strains were lower in cyclists than in controls whereas no significance difference was found for the circumferential strain. The results of our study showed that in athletes radial strain was lower than in controls, especially in the apical level and the anterior and lateral walls. We also found that circumferential strain was equal or slightly less than in controls, except for anterior and anterolateral regions at mid and apical level where it increased. Similar results were reported by Baggish et al. ${ }^{27}$ The authors hypothesized that this behavior may be due to simultaneous adaptation of the right ventricle.

Finally, it is worth mentioning that the use of our two-level acceleration registration algorithm allowed significantly lowering the time for the MRI tagging-based strain computation such as to make it a real-time technique in clinical practice. In summary, the results of this descriptive study suggest that the use of strain distribution is valuable as part of the assessment of LVH. In particular, our results show that: 1) in patients with LVH, strain distributions identified systolic dysfunction since strain was significantly reduced (lower mean); 2) HCM patients showed a larger heterogeneity (greater dispersion) in the magnitude of the strain values and presented at least one non-deforming segment, and 3) HT-LVH patients showed reduced circumferential strain, especially in the basal septum, but none of the segments were non-deforming. Thus, the use of strain distributions is a 
useful tool to evaluate myocardial abnormality in HCM patients, and to differentiate HCM from other forms of hypertrophy.

\section{Study limitations}

One limitation that may influence the strain estimation is the artifacts caused by position shift between breath-holds. This complicates the extraction of the epi- and endocardium surfaces, which are needed for defining the radial and circumferential directions. A shift correction algorithm such as the one proposed by Lötjönen et al. ${ }^{28}$ may help to circumvent this problem. In general, images with a smaller tag spacing and higher resolution or, alternatively, advance tagging techniques such as $3 \mathrm{D} \mathrm{CSPAMM}^{29}$ would need to be used to improve the strain quantification. Another potential weakness is the multiple testing which increases the risk of reporting a significant difference when there are none. However, as the $p$-values for the main results were normally below 0.001 , the chance of finding a false positive is low. Other important limitations of our clinical study include the limited size and age mismatch between healthy and pathological populations. Although our study was based primarily on deformation characterization of typical groups of patients; it would be interesting in the future to evaluate myocardial deformation in young patients with HCM and without phenotypic expression.

\section{Conclusions}

Using tagged MRI, and a fast nonrigid registration algorithm for strain quantification, allows for an easy construction of the strain distributions. The analysis of these distributions characterizes the specific pattern of myocardial deformation in patients with different etiologies of LVH. In HCM, average strain was significantly lower compared to controls, athletes and HT-LVH, and non-deforming regions were found, resulting in a larger 
heterogeneity of the strain values. These findings may provide important diagnostic information in hypertrophic diseases to establish a differential diagnostic.

\section{References}

1. Maron BJ. Hypertrophic cardiomyopathy: a systematic review. J Am Med Assoc 2002, 287:1308-1320.

2. Grossman W, Jones W, McLaurin LP. Wall stress and patterns of hypertrophy in the human left ventricle. J Clin Invest 1975, 56:56-64.

3. Maron BJ, Pelliccia A, Spirito P. Cardiac disease in young trained athletes: insights into methods for distinguishing athlete's heart from structural heart disease, with particular emphasis on hypertrophic cardiomyopathy. Circulation 1995, 91:1596-1601.

4. Vinereanu D, Florescu N, Sculthorpe N, Tweddel AC, Stephens MR, Fraser AG. Differentiation between pathologic and physiologic left ventricular hypertrophy by tissue doppler assessment of long-axis function in patients with hypertrophic cardiomyopathy or systemic hypertension and in athletes. Am J Cardiol 2001, 88:53-58.

5. Orlando S, Marciniak M, Karu T, Marciniak A, Baltabaeva A, Bijnens B, et al. Hypertrophic cardiomyopathy can be effectively discriminated from others forms by the presence of localised non-deforming segments - A deformation imaging study. Eur Heart J 2007;28 (Suppl 1).

6. Kato TS, Noda A, Izawa H, Yamada A, Obata K, Nagata K, et al. Discrimination of nonobstructive hypertrophic cardiomyopathy from hypertensive left 
ventricular hypertrophy on the basis of strain rate imaging by tissue doppler ultrasonography. Circulation 2004; 110:3808-3814.

7. Kramer CM, Reichek N, Ferrari VA, Theobald T, Dawson J, Axel L. Regional heterogeneity of function in hypertrophic cardiomyopathy. Circulation 1994; 90:186-194.

8. Serri K, Reant P, Lafitte M, Berhouet M, Le Bouffos V, Roudaut R, et al. Global and regional myocardial function quantification by two-dimensional strain: application in hypertrophic cardiomyopathy. J Am Coll Cardiol 2006; 47:1175-1181.

9. Young AA, Kramer CM, Ferrari VA, Axel L, Reichek N. Three-dimensional left ventricular deformation in hypertrophic cardiomyopathy. Circulation 1994; 90:854-867.

10. Chandrasheckara R, Mohiaddin R H, Rueckert D. Analysis of 3D myocardial motion in tagged MR images using non rigid image registration. IEEE Trans Med Imag 2004; 23:1245-50.

11. Rueckert D, Sonoda LI, Hayes C, Hill DLG, Leach MO, Hawkes DJ. Nonrigid registration using free-form deformations: application to breast MR images. IEEE Trans Med Imag 1999; 18:712-21.

12. Piella G, De Craene M, Oubel E, Larrabide I, Huguet M, Bijnens BH et al. Myocardial deformation from tagged MRI in hypertrophic cardiomyopathy 
using an efficient registration strategy. In Proceedings of SPIE, Medical Imaging (Orlando, Florida) 2009; 7262:726226-726226-10.

13. Cerqueira MD, Weissman NJ, Dilsizian V, Jacobs AK, Kaul S, Laskey WK, et al. Standardized Myocardial Segmentation and Nomenclature for Tomographic Imaging of the Heart: A Statement for Healthcare Professionals From the Cardiac Imaging Committee of the Council on Clinical Cardiology of the American Heart Association. Circulation 2002; 105:539-542.

14. Bogaert J, Rademakers FE. Regional nonuniformity of normal adult human left ventricle. Am J Physiol Heart Circ Physiol 2001; 280:H610-H620.

15. Moore CC, Lugo-Olivieri CH, McVeigh ER, Zerhouni EA. Three-dimensional systolic strain patterns in the normal human left ventricle: characterization with tagged MR imaging. Radiology 2000; 214:453-466.

16. Van Rugge FP, Holman ER, Wall EE, Roos A, Laarse A, Bruschke AG. Quantification of global and regional left ventricular function by cine magnetic resonance imaging during dobutamine stress in normal human subjects. Eur Heart J 1993; 14:456-463.

17. Varnava AM, Elliott PM, Sharma S, McKenna WJ, Davies MJ. Hypertrophic cardiomyopathy: the interrelation of disarray, fibrosis, and small vessel disease. Heart 2000; 84:476-482. 
18. St John Sutton MG, Lie JT, Anderson KR., O’Brien PC, Frye RL.

Histopathological specificity of hypertrophic obstructive cardiomyopathy. Myocardial fibre disarray and myocardial fibrosis. Br Heart J 1980; 44:433-443.

19. Kim Y, Choi BY, Hur J, Lee HJ, Seo JS, Kim TH et al. Delay enhancement in hypertrophic cardiomyopathy: comparison with myocardial tagging MRI. $J$ Magn Reson Imag 2008; 27:1054-1060.

20. Rajiv D, Fraser AG. Tissue Doppler imaging for the evaluation of patients with hypertrophic cardiomyopathy. Curr Opin Cardiol 2004; 19:430 - 436.

21. Ganau A, Devereux, RB, Roman MJ, de Simone G, Pickering TG, Saba PS, et al. Patterns of left ventricular hypertrophy and geometric remodeling in essential hypertension. J Am Coll Cardiol 1992; 19:1550-1558.

22. Baltabaeva A, Marciniak M, Bijnens B, Moggridge J, He FJ, Antonios TF, et al. Regional left ventricular deformation and geometry analysis provides insights in myocardial remodelling in mild to moderate hypertension. Eur $J$ Echocardiogr 2008; 9:501-508.

23. Maron BJ. Structural features of the athlete heart as defined by echocardiography. J Am Coll Cardiol 1986; 7:190-203.

24. Petersen SE, Selvanayagam JB, Francis JM, Myerson SG, Wiesmann F, Robson MD, et al. Differentiation of athlete's heart from pathological forms of cardiac hypertrophy by means of geometric indices derived from cardiovascular magnetic resonance. J Cardiovasc Magn Reson 2005; 7:551-558. 
25. Richand V, Lafitte S, Reant P, Serri K, Lafitte M, Brette S, et al. An ultrasound speckle tracking (two-dimensional strain) analysis of myocardial deformation in professional soccer players compared with healthy subjects and hypertrophic cardiomyopathy. Am J Cardiol 2007; 100:128 - 132.

26. Nottin S, Doucende G, Schuster-Beck I, Dauzat M, Obert P. Alteration in left ventricular normal and shear strains evaluated by 2D-strain echocardiography in the athlete's heart. $J$ Physiol 2008; 586:4721-4733.

27. Baggish A, Yared K, Wang F, Weiner RB, Hutter AM, Picard MH et al. The impact of endurance exercise training on left ventricular systolic mechanics. Am J Physiol Heart Circ Physiol 2008; 295: H1109-H1116.

28. Lötjönen J, Pollari M, Kivistö S, Lauerma K. Correction of Movement Artifacts from 4-D Cardiac Short and Long-Axis MR Data. In Proceedings of the 7th International Conference on Medical Image Computing and Computer-Assisted Intervention, (MICCAI'04), Part II. Edited by Barillot C, Haynor DR, Hellier P. vol. 3217 of Lect Notes in Comput Sci:405-412, Springer 2004.

29. Rutz A, Ryf S, Plein S, Boesiger P, Kozerke S. Accelerated whole-heart 3D CSPAMM for myocardial motion quantification. Magn Reson Med 2008, 59:755-763. 


\section{Tables}

Table 1 - Baseline characteristics of the studied population. Results are expressed as mean \pm standard deviation

\begin{tabular}{|c|c|c|c|c|}
\hline Variables & $\begin{array}{l}\text { Controls } \\
(n=12) \\
\end{array}$ & $\begin{array}{l}\text { Athletes } \\
(n=10)\end{array}$ & $\begin{array}{l}\mathrm{HCM} \\
(\mathrm{n}=12)\end{array}$ & $\begin{array}{l}\text { HT-LVH } \\
(n=10)\end{array}$ \\
\hline Age & $28.7 \pm 2.7$ & $23.2 \pm 3.0$ & $61.4 \pm 14.1$ & $65.5 \pm 16.0$ \\
\hline$\overline{\text { Sex (male:female) }}$ & $8: 4$ & $10: 0$ & $8: 4$ & $6: 4$ \\
\hline LV ejection fraction (\%) & $61.1 \pm 4.2$ & $61.3 \pm 6.5$ & $68.9 \pm 7.6$ & $63.5 \pm 11.8$ \\
\hline LV end-diastolic diameter (mm) & $48.0 \pm 3.7$ & $57.5 \pm 2.8$ & $43.8 \pm 5.9$ & $51.1 \pm 3.6$ \\
\hline LV end-systolic diameter $(\mathrm{mm})$ & $35.5 \pm 3.9$ & $41.8 \pm 3.4$ & $26.4 \pm 8.0$ & $27.8 \pm 1.94$ \\
\hline LV end-diastolic volume (ml) & $124 \pm 25.4$ & $226.2 \pm 47.6$ & $127.4 \pm 37.6$ & $135.0 \pm 23.3$ \\
\hline$\overline{\mathrm{LV}} \overline{\text { end-systolic volume (ml) }}$ & $48.1 \pm 11.5$ & $85.6 \pm 13.1$ & $41.1 \pm 16.4$ & $50.6 \pm 21.2$ \\
\hline$\overline{\mathrm{LV}} \overline{\text { septal wall thickness (mm) }}$ & $8.4 \pm 1.1$ & $11.7 \pm 1.5$ & $20.7 \pm 3.6$ & $14.5 \pm 2.0$ \\
\hline LV posterior wall thickness $(\mathrm{mm})$ & $8.4 \pm 1.4$ & $10.7 \pm 1.3$ & $9.6 \pm 1.7$ & $8.7 \pm 1.9$ \\
\hline Maximum LV wall thickness (mm) & $8.4 \pm 1.5$ & $11.7 \pm 1.5$ & $20.7 \pm 3.6$ & $15.1 \pm 1.4$ \\
\hline Ratio septum/posterior wall & $1.0 \pm 0.1$ & $1.1 \pm 0.1$ & $2.1 \pm 0.5$ & $1.7 \pm 0.4$ \\
\hline \multicolumn{5}{|l|}{ thickness } \\
\hline LV mass $(\mathrm{g})$ & $99.8 \pm 40$ & $167.5 \pm 16.2$ & $180.5 \pm 43.0$ & $130.9 \pm 23.3$ \\
\hline LV mass index $(\%)$ & $55.2 \pm 21.0$ & $89.8 \pm 5.8$ & $98.0 \pm 31.9$ & $70.3 \pm 12.8$ \\
\hline
\end{tabular}


Table 2 - Radial mean peak systolic strains in the studied population. Results are expressed as mean \pm standard deviation

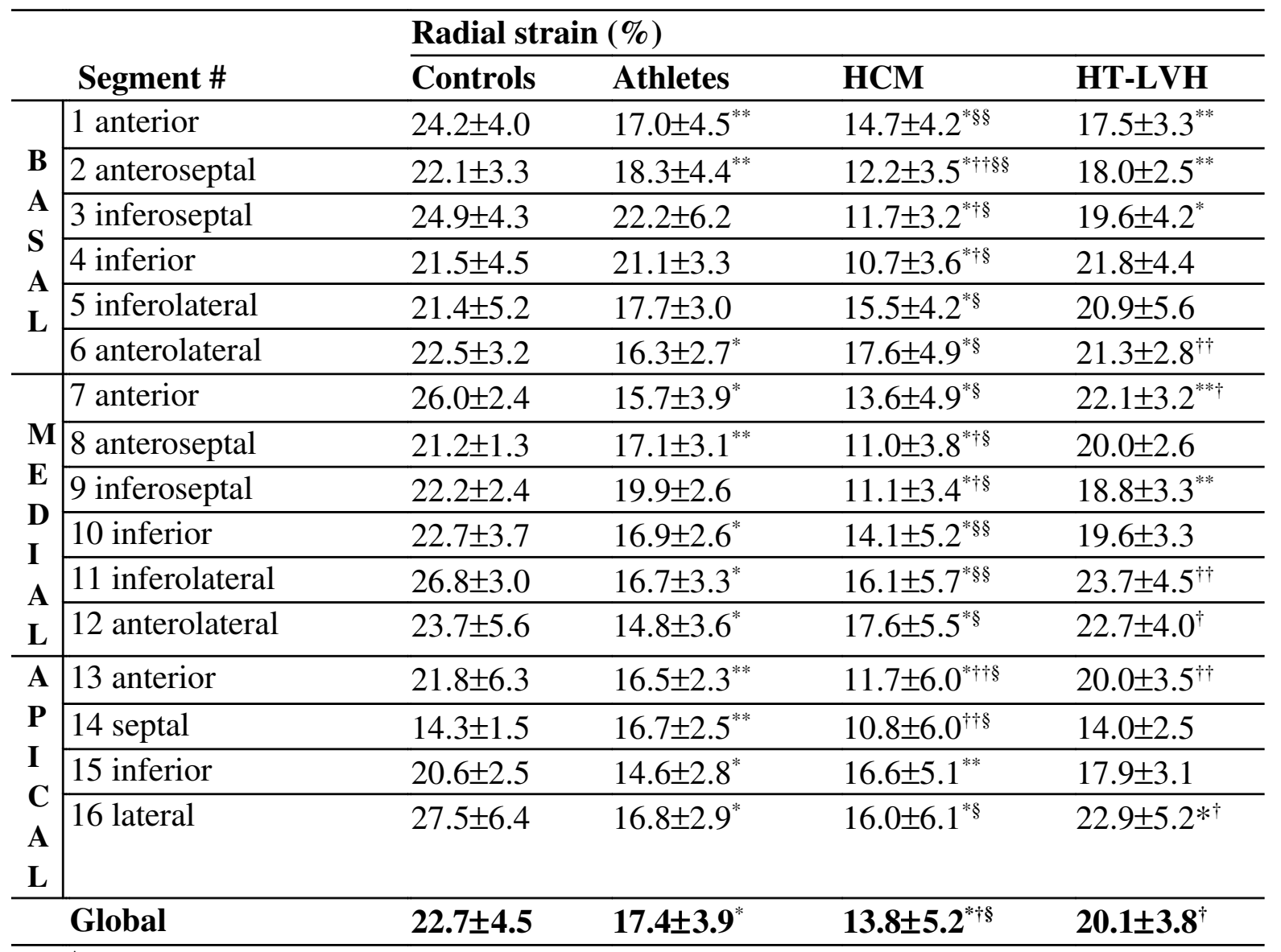

${ }^{*} \mathrm{p}<0.001$ when comparing with controls

${ }^{* *} \mathrm{p}<0.05$ when comparing with controls

${ }^{\dagger} \mathrm{p}<0.001$ when comparing with athletes

${ }^{\dagger} \mathrm{p}<0.05$ when comparing with athletes

${ }^{\S} \mathrm{p}<0.001$ when comparing with HT-LVH

$\S \S \mathrm{p}<0.05$ when comparing with HT-LVH 
Table 3 -Circumferential mean peak systolic strains in the studied population.

Results are expressed as mean \pm standard deviation

\begin{tabular}{|c|c|c|c|c|c|}
\hline & & $\overline{\text { Circumfe }}$ & tial strain (c & & \\
\hline & Segment \# & Controls & Athletes & HCM & HT-LVH \\
\hline $\mathbf{B}$ & 1 anterior & $-11.5 \pm 1.1$ & $-11.8 \pm 2.0$ & $-8.8 \pm 2.9^{* *+\dagger \S \S}$ & $-11.5 \pm 1.4$ \\
\hline $\mathbf{A}$ & 2 anteroseptal & $-12.1 \pm 0.5$ & $-11.1 \pm 1.8$ & $-10.0 \pm 2.8^{* * *}$ & $-8.0 \pm 1.8^{* \dagger}$ \\
\hline $\mathbf{S}$ & 3 inferoseptal & $-15.1 \pm 2.5$ & $-11.5 \pm 3.0^{* * *}$ & $-7.9 \pm 2.6^{* \neq \dagger}$ & $-8.2 \pm 2.5^{* \dagger \dagger}$ \\
\hline $\mathbf{A}$ & 4 inferior & $-12.4 \pm 1.4$ & $-11.9 \pm 2.2$ & $-6.9 \pm 2.4^{*+8}$ & $-10.1 \pm 2.1$ \\
\hline & 5 inferolateral & $-12.8 \pm 1.5$ & $-12.5 \pm 1.9$ & $-9.2 \pm 2.7^{* * \dagger \S}$ & $-11.1 \pm 2.4$ \\
\hline & 6 anterolateral & $-11.8 \pm 1.1$ & $-11.8 \pm 2.4$ & $-8.6 \pm 3.4^{* * \dagger \dagger}$ & $-10.5 \pm 3.0$ \\
\hline & 7 anterior & $-12.6 \pm 0.7$ & $-14.9 \pm 1.8^{* * *}$ & $-10.1 \pm 4.0^{\dagger \dagger}$ & $-11.4 \pm 3.2$ \\
\hline $\mathbf{M}$ & 8 anteroseptal & $-14.8 \pm 0.8$ & $-14.7 \pm 1.1$ & $-10.9 \pm 4.0^{* *+\dagger}$ & $-11.1 \pm 3.2^{* \dagger}$ \\
\hline $\mathbf{E}$ & 9 inferoseptal & $-14.9 \pm 2.0$ & $-12.5 \pm 2.4^{* *}$ & $-9.6 \pm 2.8^{* \dagger \dagger}$ & $-11.9 \pm 2.4^{*}$ \\
\hline $\mathbf{I}$ & 10 inferior & $-14.4 \pm 2.5$ & $-14.1 \pm 2.2$ & $-9.4 \pm 2.7^{*+\S}$ & $-13.6 \pm 2.5$ \\
\hline $\mathbf{A}$ & 11 inferolateral & $-15.1 \pm 1.7$ & $-14.4 \pm 1.4$ & $-12.6 \pm 3.2^{* *}$ & $-14.3 \pm 2.6$ \\
\hline $\mathbf{L}$ & 12 anterolateral & $-12.6 \pm 1.8$ & $-16.1 \pm 1.8^{*}$ & $-10.7 \pm 3.9^{\dagger}$ & $-12.3 \pm 3.2$ \\
\hline $\mathbf{A}$ & 13 anterior & $-13.4 \pm 1.2$ & $-15.9 \pm 2.2^{* * *}$ & $-7.0 \pm 3.4^{* \dagger}$ & $-10.0 \pm 3.1^{\dagger}$ \\
\hline $\mathbf{P}$ & 14 septal & $-14.5 \pm 2.1$ & $-13.8 \pm 2.1$ & $-8.5 \pm 4.0^{* \dagger}$ & $-12.5 \pm 2.9$ \\
\hline I & 15 inferior & $-14.4 \pm 2.3$ & $-15.2 \pm 3.2$ & $-9.5 \pm 2.8^{* \dagger}$ & $-12.7 \pm 3.1$ \\
\hline A & 16 lateral & $-14.7 \pm 1.6$ & $-15.3 \pm 2.8$ & $-8.2 \pm 3.4^{*+\S}$ & $-12.2 \pm 2.2^{* \dagger}$ \\
\hline $\mathbf{L}$ & & & & & \\
\hline & Global & $-13.6 \pm 2.2$ & $-13.6 \pm 2.9$ & $-9.2 \pm 3.4^{* \dagger \S \S}$ & $-11.3 \pm 3.1^{* *+\dagger}$ \\
\hline & $\begin{array}{l}{ }^{*} \mathrm{p}<0.001 \text { when } \\
{ }^{*} \mathrm{p}<0.05 \text { when } \\
{ }^{+} \mathrm{p}<0.001 \text { wher } \\
{ }^{\dagger} \mathrm{p}<0.05 \text { when }<0.001 \text { wher } \\
{ }^{8} \mathrm{p}<0.05 \text { when }\end{array}$ & $\begin{array}{l}\text { ring with } \\
\text { ring with c } \\
\text { aring with } \\
\text { ring with a } \\
\text { aring with } \\
\text { ring with } \mathrm{F}\end{array}$ & $\begin{array}{l}\text { rols } \\
\text { ols } \\
\text { tes } \\
\text { tes } \\
\text { LVH } \\
\text { LVH }\end{array}$ & & \\
\hline
\end{tabular}


Table 4 -Dispersion of radial peak systolic strain in the studied population. The reported values are the average of the radial strain dispersion values of each

individual within the group. Results are expressed as mean \pm standard deviation.

\begin{tabular}{lllll}
\hline \multirow{2}{*}{ Dispersion measure } & \multicolumn{4}{l}{ Radial strain dispersion } \\
\cline { 2 - 5 } & Controls & Athletes & HCM & HT-LVH \\
\hline CV(\%) & $21.0 \pm 2.2$ & $19.6 \pm 2.9$ & $\mathbf{2 9 . 7} \pm \mathbf{5 . 3 ^ { * * \S }}$ & $21.0 \pm 5.0$ \\
\hline Range & $\mathbf{1 7 . 7} \pm \mathbf{1 . 5}$ & $11.0 \pm 2.0^{*}$ & $15.8 \pm 3.8^{*+\S}$ & $17.7 \pm 2.1^{\dagger}$ \\
\hline IQR & $\mathbf{6 . 1} \pm \mathbf{1 . 2}$ & $4.8 \pm 1.2^{*}$ & $5.7 \pm 1.8^{*}$ & $5.8 \pm 1.6^{*}$ \\
\hline
\end{tabular}

$* p<0.01$ when comparing with controls

${ }^{\dagger} \mathrm{p}<0.01$ when comparing with athletes

${ }^{\S} \mathrm{p}<0.01$ when comparing with HT-LVH 
Table 5 -Dispersion of circumferential peak systolic strain in the studied population. The reported values are the average of the circumferential strain dispersion values of each individual within the group. Results are expressed as mean \pm standard deviation.

\begin{tabular}{lllll}
\hline \multirow{2}{*}{ Dispersion measure } & \multicolumn{4}{l}{ Circumferential strain dispersion } \\
\cline { 2 - 5 } & Controls & Athletes & HCM & HT-LVH \\
\hline CV(\%) & $15.4 \pm 3.4$ & $17.8 \pm 4.7$ & $\mathbf{3 1 . 7 \pm 9 . 0 ^ { * + \S }}$ & $22.1 \pm 1.9^{*}$ \\
\hline Range & $6.6 \pm 1.9$ & $8.9 \pm 1.3^{*}$ & $\mathbf{1 0 . 1} \pm \mathbf{2 . 0}{ }^{*}$ & $8.7 \pm 2.4^{*}$ \\
\hline IQR & $3.4 \pm 0.8$ & $3.8 \pm 0.9$ & $\mathbf{3 . 9} \pm \mathbf{1 . 1}$ & $3.8 \pm 1.0$ \\
\hline
\end{tabular}

" $\mathrm{p}<0.01$ when comparing with controls

p $<0.01$ when comparing with athletes

${ }^{\S} \mathrm{p}<0.01$ when comparing with HT-LVH 
Table 6 - Presence of non-deforming (and deforming) segments within late gadolinium enhancement and non late gadolinium enhancement segments.

\begin{tabular}{lll}
\hline & LGE $(\mathbf{n}=\mathbf{3 0})$ & Non LGE $(\mathbf{n}=\mathbf{1 5 3})$ \\
\hline Non-deforming & $60 \%$ & $11.8 \%$ \\
Deforming & $40 \%$ & $88.2 \%$ \\
\hline
\end{tabular}


Table 7 - Presence of late gadolinium enhancement (and non late gadolinium enhancement) segments within non-deforming and deforming segments

\begin{tabular}{lll}
\hline & Non-deforming $(\mathbf{n}=\mathbf{3 6})$ & Deforming $(\mathbf{n}=\mathbf{1 4 7})$ \\
\hline LGE & $50 \%$ & $8.2 \%$ \\
Non LGE & $50 \%$ & $91.8 \%$ \\
\hline
\end{tabular}




\section{FIGURES}
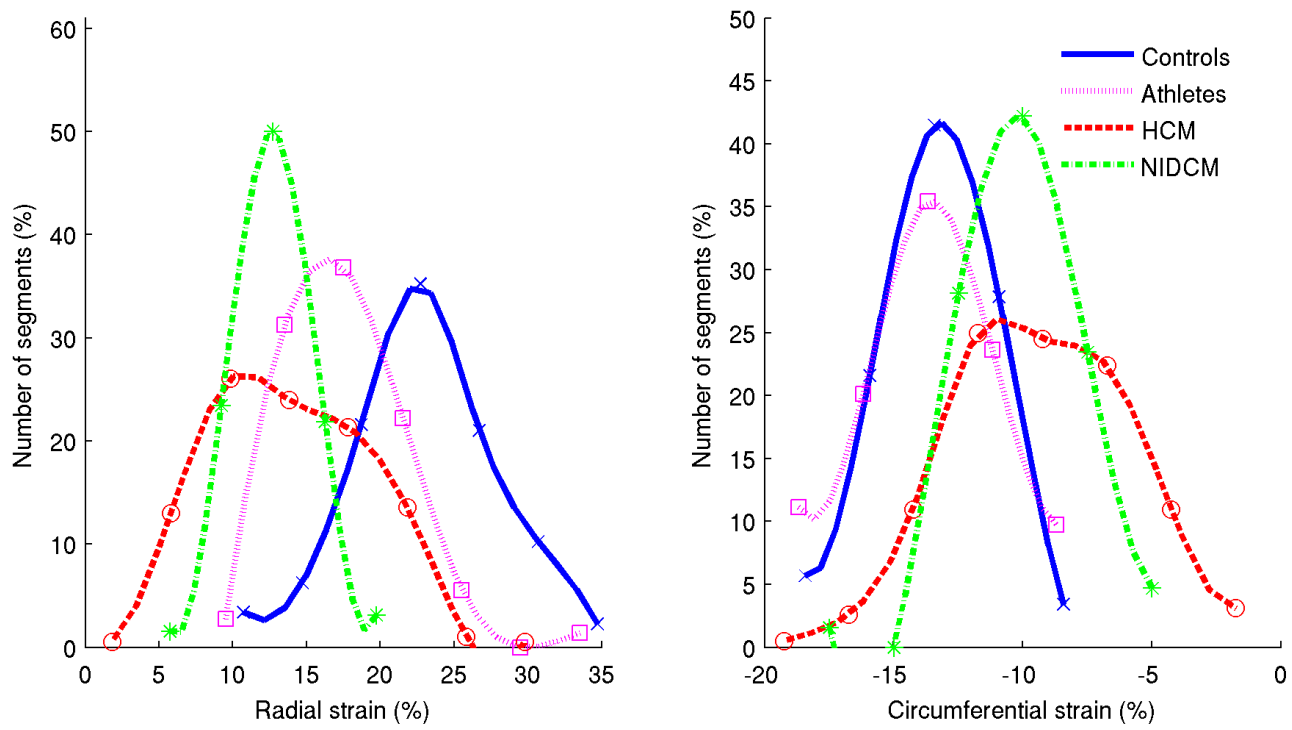

Figure 1. Distribution of radial (left) and circumferential (right) peak systolic strain in control group (blue solid line), athletes (magenta dotted line), HCM group (red dashed line) and HT-LVH group (green dot-dashed line). Positive values indicate thickening while negative values indicate thinning. The significant higher spread in HCM results from the coexistence of segments with impaired deformation and segments with normal contraction. 

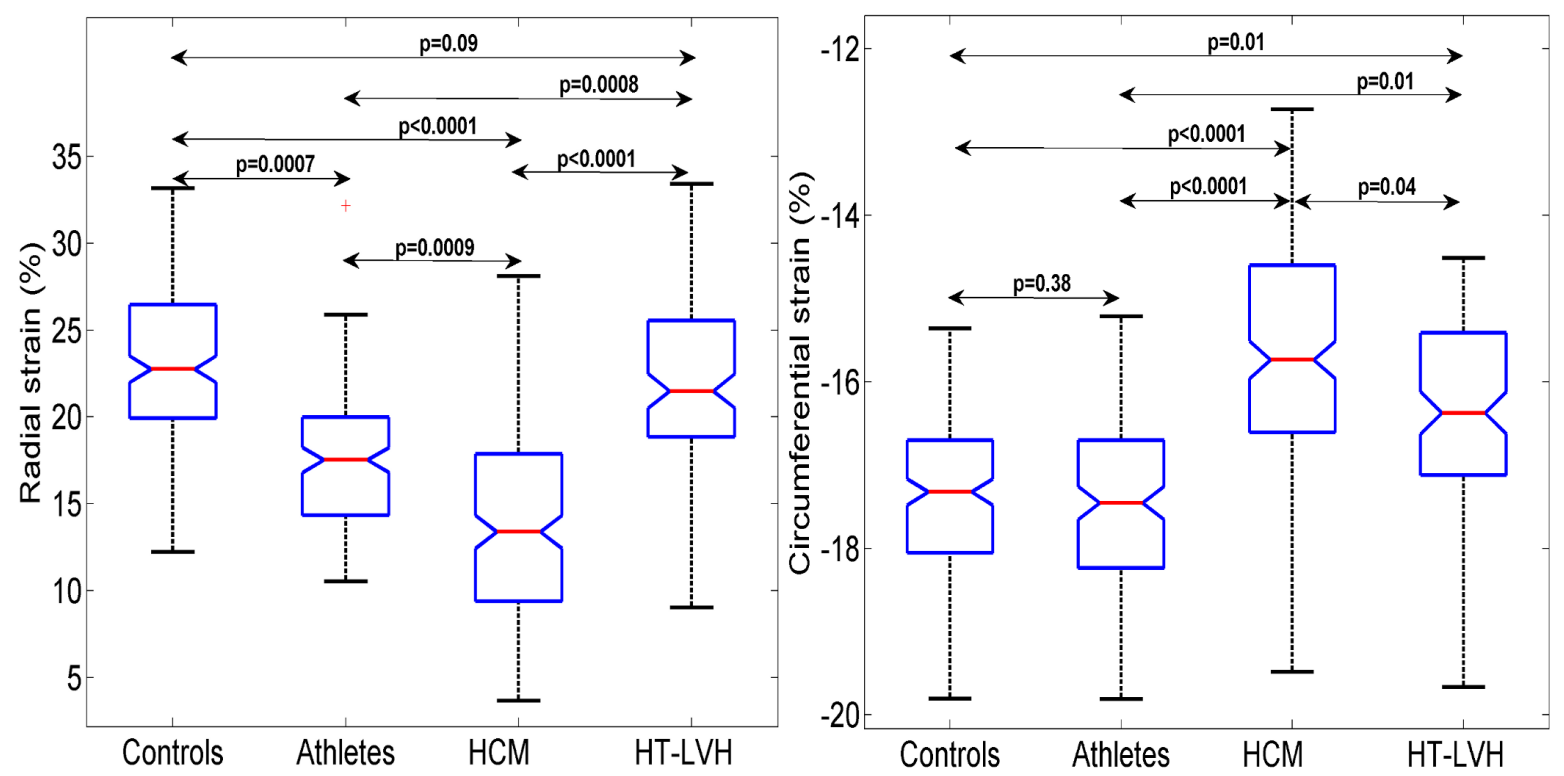

Figure 2

Boxplot of the radial (left) and circumferential (right) peak systolic strain distributions in the different study groups. Positive values indicate thickening while negative values indicate thinning. HCM group had the lowest median strain and the largest spread of strain values. 


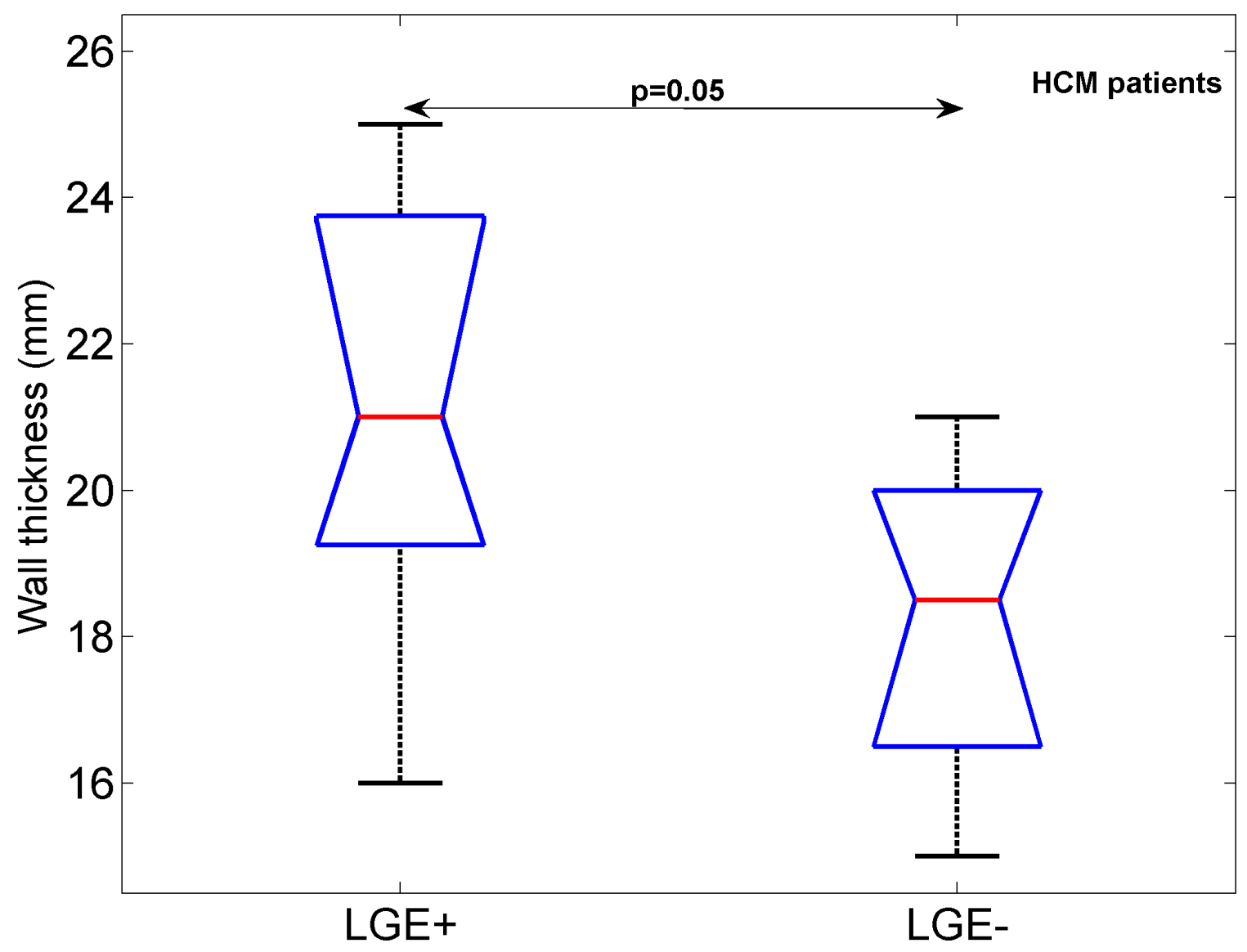

Figure 3

Boxplot showing the relation between the maximum end diastolic wall thickness and LGE in the HCM group. Patients with LGE had higher wall thickness. 